\title{
A study of working fluids for transcritical pumped thermal energy storage cycles
}

\author{
$1^{\text {st Antoine Koen }}$ \\ Department of Engineering \\ University of Cambridge \\ Cambridge, United Kingdom \\ ajk207@cam.ac.uk
}

\author{
$2^{\text {nd }}$ Pau Farres Antunez \\ Department of Engineering \\ University of Cambridge \\ Cambridge, United Kingdom \\ pf298@cam.ac.uk
}

\author{
$3^{\text {rd }}$ Alexander White \\ Department of Engineering \\ University of Cambridge \\ Cambridge, United Kingdom \\ ajw36@cam.ac.uk
}

\begin{abstract}
Electricity storage is widely regarded as critical to a sustainable energy future, and currently deployed technologies such as pumped hydroelectric storage have drawbacks which limit the scale to which they can be implemented. Pumped thermal energy storage (PTES) has recently started to attract interest as an alternative. This article focuses on transcritical cycles and aims to identify the best working fluids, in a configuration with a single hot store and no cold store. Three different storage media were considered for the hot store: water, Therminol D12, and Therminol 66. For the transcritical cycle, 176 different working fluids were screened for thermodynamic, environmental and safety suitability, and the resulting list of 8 fluids was tested with cycles at a range of storage temperatures. The optimal round-trip efficiency is a trade-off between heat exchanger losses and turbomachinery losses. Pareto fronts were used to rank the fluids for efficiency, power density, and heat to work ratio. The most promising fluid was found to be trifluoroiodomethane (R13I1) with a peak round-trip efficiency of $57.6 \%$.
\end{abstract}

Index Terms-pumped thermal energy storage, working fluid, transcritical cycle

\section{INTRODUCTION}

Renewables have seen strong growth in the last few years and are predicted to continue expanding. However, reaching substantial levels of penetration on the grid is challenging and will require among other things large scale storage technologies to compensate for intermittency. At present, the options for storage that are compatible with the energy trilemma (affordability, security, and sustainability) are limited, and each has its own characteristics which restrict it or suit it to some applications better than others. Most notably, pumped hydro storage (PHS) and compressed air energy storage (CAES) are inherently site-dependent. Pumped thermal energy storage (PTES) consists of storing electricity as heat with a reversible heat pump, which has the advantage of being site-independent and potentially highly cost-effective, since the storage medium can be very cheap and with high energy density, as can be seen in Table I. This medium could be water, concrete, or other fluids and solids. Many variants of PTES are being investigated. This work focuses on a configuration for sensible heat storage (as opposed to latent heat) with a liquid storage material.

The first author gratefully acknowledges the UK Engineering and Physical Sciences Research Council (EPSRC) for its sponsorship enabling this study.

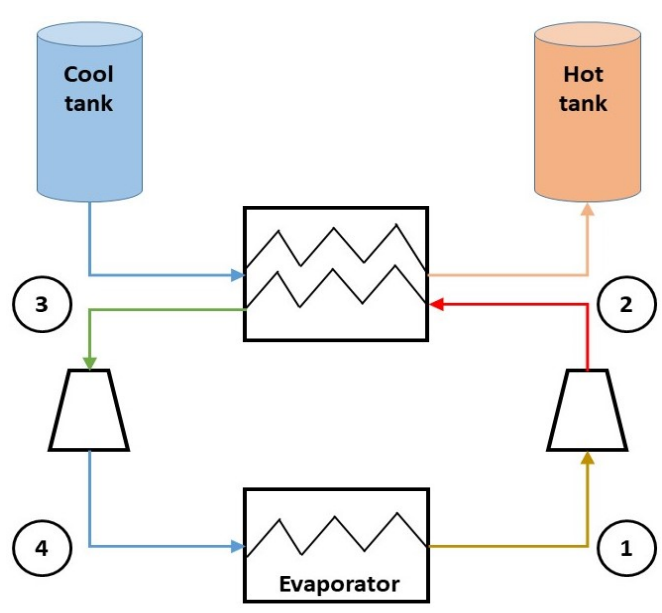

Fig. 1. Cycle points and components, shown during charge (during discharge, the arrows are reversed and the evaporator is a condenser)

TABLE I

COMPARISON OF STORAGE TECHNOLOGIES, FROM [1]

\begin{tabular}{lccc}
\hline & PHS & CAES & PTES \\
\hline Working fluid & Water & Air & Argon \\
Storage medium & Water & Air & Gravel \\
Energy density $\left(\mathrm{kWh} / \mathrm{m}^{3}\right)$ & 1.4 & 10 & 50 \\
Power density $\left(\mathrm{kW} / \mathrm{m}^{3} \cdot \mathrm{s}^{-1}\right)$ & 5000 & - & 240 \\
\hline
\end{tabular}

Figure 1 shows the basic layout of a PTES system: a working fluid runs a thermodynamic cycle with an expander, compressor, heat exchanger, and evaporator/condenser, while the storage fluid flows from a cold tank to a hot tank, receiving heat from the working fluid in between.

Three main thermodynamic cycles exist for the reversible heat pump: Rankine, Joule-Brayton, and transcritical (Fig. 2). For each, the following two metrics help to quantify the suitability of these cycles (and the associated working fluids) for storage systems.

i) The work ratio is the amount of compression work (points 1 to 2 ) divided by the expansion work performed during charge (points 3 to 4 ). A high ratio will reduce the losses in round-trip efficiency due to the turbomachinery and is therefore desirable.

ii) The heat storage ratio is the amount of heat stored 


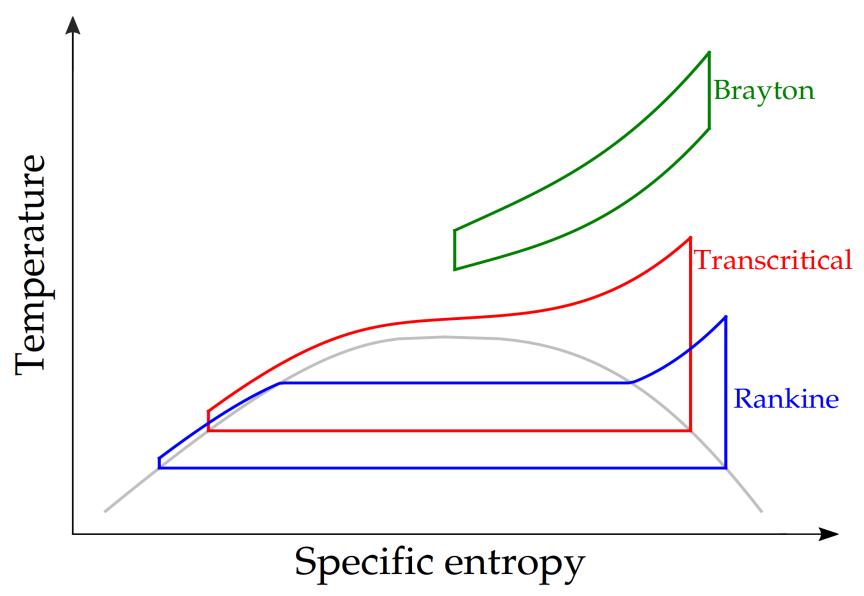

Fig. 2. Brayton, Rankine and transcritical cycles on a T-s diagram

TABLE II

TYPICAL VALUES OF HEAT STORAGE RATIO AND WORK RATIO, FROM [2]

\begin{tabular}{lcc}
\hline Cycle & Heat storage ratio & Work ratio \\
\hline Rankine & 5 & 20 \\
Transcritical & 2.7 & 5 \\
Brayton & 1.1 & 2.2 \\
\hline
\end{tabular}

(points 2 to 3 ) divided by the compression work during charge. A high ratio requires more storage material and heat exchanger surface area per unit work stored and is therefore undesirable.

The transcritical cycle is attractive because it combines the benefits of the Brayton cycle (low heat storage ratio) with those of the Rankine cycle (high work ratio, because expanding a liquid produces less work than for a gas), as can be seen in Table II.

The vast majority of proposed transcritical cycles run on carbon dioxide [2]-[7]. The reasons usually cited are that carbon dioxide is low cost, widely available, safe to handle and has low impact on the environment, with high density which allows for a more compact and hence cheaper system. However, to the best knowledge of the authors, no actual cycle models have been published directly comparing the performance of various fluids for transcritical PTES. Note that the transcritical cycle studied herein bottoms at ambient temperature and thus differs slightly from the literature on transcritical carbon dioxide, for which models usually include a cold store.

\section{FLUID SELECTION}

An initial list of 176 candidate working fluids was compiled, dictated by the ones available in the thermophysical libraries CoolProp and Refprop. Many of them are refrigerants, but the list also includes many common fluids like air, carbon dioxide, or butane.

\section{A. Thermodynamics}

The majority of these fluids are not suitable for a transcritical cycle bottoming at ambient temperature $\left(T_{0} \sim 15^{\circ} \mathrm{C}\right)$. The minimum following conditions must be met:

i) $T_{\text {crit }}>T_{0}$

This is a thermodynamically necessary condition, since the cycle is rejecting heat at ambient during discharge.

ii) $P_{\text {crit }}<250$ bar

This is a technical requirement: the pressures reached in the cycle must be realistically manageable from a materials point of view. Setting the limit at $250 \mathrm{bar}$ is informed by the experimental setup in [8] which reaches 200 bar, while their design calculations go up to 250 bar. (Supercritical turbines with inlet pressures of 290 bar are also standard in coal-fired generation, with the industry seeking even higher pressures up to 350 bar in future [9].)

This narrows the list down to 84 wet fluids and 24 dry fluids. A wet fluid has a saturation vapour curve with a negative slope on the T-s diagram, whereas a dry fluid's saturation vapour curve has a positive slope. This difference in shape will affect how much the fluid enters the two-phase region during compression (during charge) and expansion (during discharge). This consideration will be developed further later on in section IV.

\section{B. Environment}

Ever since the Montreal protocol, there are strict regulations concerning refrigeration fluids (and therefore many in the aforementioned list) in terms of their ozone depletion potential (ODP). More recent regulations restrict the use of fluids in terms of their global warming potential (GWP) as well. The most stringent European Union regulatory guidelines are used here to discriminate between the fluids: zero or negligible ozone depletion potential (below around 0.02 [10]) and low global warming potential (below 150 [10]). Following a precautionary principle in this way ensures that the resulting list of fluids is immune to potential phase-out in the future and thus renders the environmental acceptability of these fluids much more certain. Several databases [10]-[13] were used to manually check the environmental acceptability of the preselected fluids.

This narrows the list down to 19 wet fluids (acetone, ammonia, carbon dioxide, carbonyl sulfide, cyclopropane, dimethyl ether, ethane, ethylene oxide, hydrogen chloride, hydrogen sulfide, propylene, propyne, R13I1, R152A, R161, R41, sulfur dioxide, xenon, propane) and 10 dry fluids (butene, isobutane, isobutene, neopentane, R1234yf, R1234ze(E), R1234ze(Z), cis-2-butene, butane, trans-2-butene). Xenon was eliminated due to its critical temperature being very close to ambient $\left(T_{\text {crit }}=16.58^{\circ} \mathrm{C}\right)$, which would make the cycle highly sensitive to changes in ambient temperature and even impossible to run at some times of the year and hours of the day (if $\left.T_{\text {crit }}<T_{a m b}\right)$. Note that these 28 remaining substances all have vapour pressures at ambient temperature above 1 bar (except for acetone at 0.2 bar), which is useful for avoiding air infiltration from the outside into the working fluid, which would degrade its performance. 


\section{Safety}

Ideally, the working fluid of a PTES system should also be safe to handle. However, few fluids are totally harmless, so the question becomes what constitutes acceptable risk for industry. Most of the selected fluids are highly flammable, and therefore only the safest ones or those currently used as refrigerants (and therefore that can be handled with confidence) were kept: ammonia, carbon dioxide, R13I1, R152A, R161, R1234yf, $\mathrm{R} 1234 \mathrm{ze}(\mathrm{E}), \mathrm{R} 1234 \mathrm{ze}(\mathrm{E})$. The first five are wet fluids, the remaining three dry fluids.

\section{MODEL}

\section{A. Storage material}

TABLE III

STORAGE MEDIA AT AMBIENT PRESSURE

\begin{tabular}{cc}
\hline Maximum storage temperature & Storage medium \\
\hline $100^{\circ} \mathrm{C}$ & Water \\
$192^{\circ} \mathrm{C}$ & Therminol D12 \\
$359^{\circ} \mathrm{C}$ & Therminol 66 \\
\hline
\end{tabular}

Together, the materials in Table III provide a low to medium range of potential storage temperatures to explore in order to find if there is a particular value at which performance is maximised for each working fluid. The maximum storage temperature is limited by the boiling point of the storage medium, and the storage tanks are considered unpressurised for simplicity as well as cost reduction. Furthermore, only one storage medium is used for a given cycle, i.e. the heat is not stored in several materials at different temperatures but rather in a single one at a given temperature. There are therefore only two storage tanks (one is full after charge, the other after discharge), as in Fig. 1.

\section{B. Compression and expansion}

The compressions and expansions (one each for charge and discharge respectively) are modelled with a polytropic efficiency of $90 \%$.

In the turbomachinery, the exergy loss per unit mass of working fluid is:

$$
b_{\text {loss }}=T_{0}\left(s_{\text {out }}-s_{\text {in }}\right)
$$

and expressed as a fraction of the net cycle specific work during charge.

\section{Heat exchanger}

The heat exchanger is modelled with the so-called effectiveness-NTU method, as in [14]. An effectiveness of $95 \%$ is used to define a UA value for the heat exchanger, and then the outlet temperatures are determined from the inlet temperatures by iteration until convergence.

Drawing the control volume around the whole heat exchanger gives:

$$
\dot{S}_{\text {irrev }}=\dot{m}_{\text {hot }}\left(s_{\text {out }}-s_{\text {in }}\right)_{h o t}+\dot{m}_{\text {cold }}\left(s_{\text {out }}-s_{\text {in }}\right)_{\text {cold }}
$$

The specific exergy loss per unit mass of working fluid can then be computed as (pressure losses are not included):

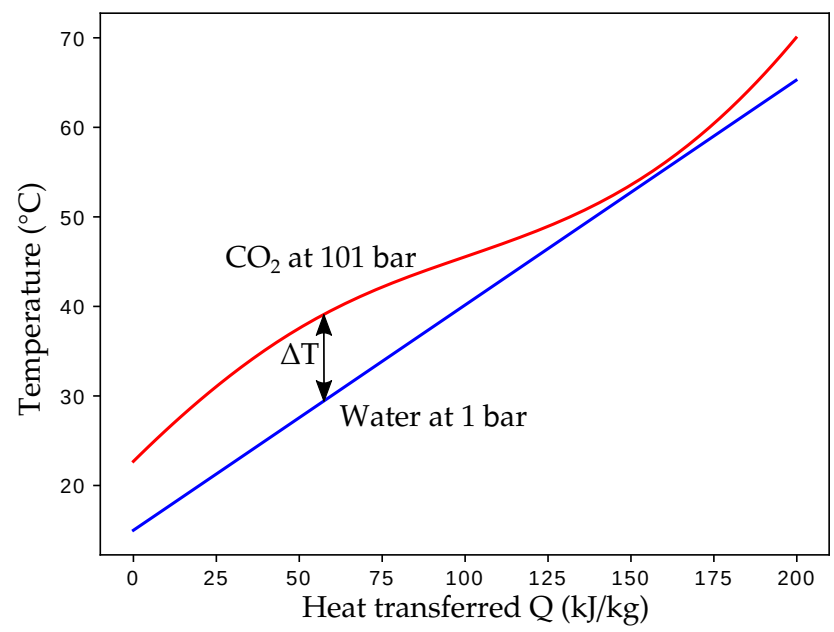

Fig. 3. T-Q diagram of the heat exchanger, with carbon dioxide and water

$$
b_{\text {loss }}=\frac{T_{0} \dot{S}_{i r r e v}}{\dot{m}_{w f}}
$$

This loss is then given as a fraction of the net cycle work during charge.

The performance of the heat exchanger is a crucial part of a transcritical cycle. Near the critical point the heat capacity of the working fluid will vary drastically, causing large temperature differences and thus irreversible entropy generation, as can be seen in Fig. 3. Matching the capacitance rates of the working and storage fluids reduces this, but also has the consequence that, for a constant mass flowrate for the working fluid during charge and discharge, the flowrate of the storage fluid has to change between charge and discharge to keep the capacitance rates matched. This means the duration of charge and discharge are not equal. This is taken into account when calculating round-trip efficiency (see equation 5).

\section{Condenser/evaporator}

The exergy loss in the condenser/evaporator is:

$$
b_{\text {loss }}=\frac{T_{0} \dot{S}_{\text {irrev }}}{\dot{m}_{w f}}=\frac{1}{\dot{m}_{w f}} \int\left(1-\frac{T_{0}}{T}\right) d \dot{Q}
$$

with the integral running from inlet to outlet i.e. from point 4 to 1 during charge and from $1^{\prime}$ to $4^{\prime}$ during discharge. The loss is then given as a fraction of the net cycle specific work during charge.

\section{E. Charging}

The only input variable is the top temperature $T_{2}$ (see Fig. 4). Point 1 is set as saturated vapour. A polytropic compression to $T_{2}$ gives point 2 . The working fluid then heats the storage fluid in the heat exchanger and cools to point 3 . The inlet temperature of the storage fluid is not known a priori but rather found by iteration such that its value is the same before charge and after discharge. If its temperature after discharge were lower than initially before charge, then the cycle could 


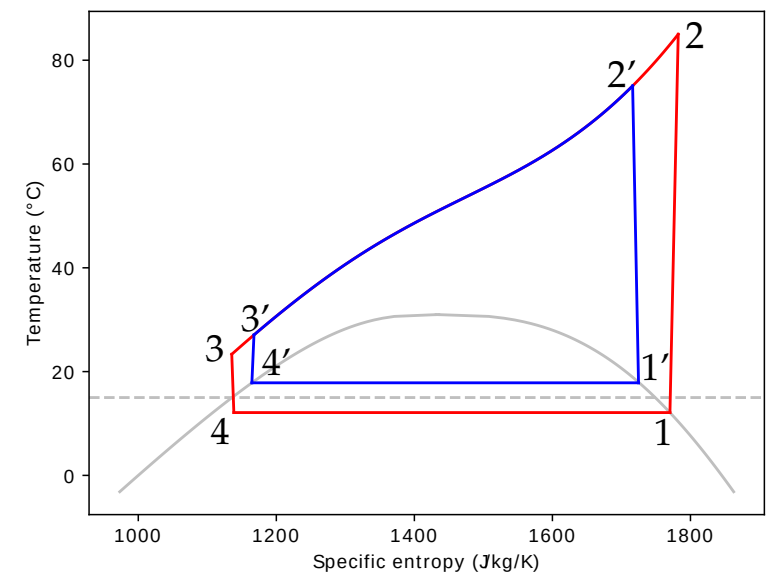

Fig. 4. Temperature-entropy diagram of the transcritical carbon dioxide charge and discharge cycles (red and blue respectively). In an ideal reversible system, the two would overlap.

not be sustainably repeated. At the outlet of the heat exchanger, the working fluid is expanded polytropically to point 4 , which is at the same pressure as point 1 and can lie outside or inside the saturation curve. The working fluid is then evaporated back to point 1 .

The location of point 1 depends on the temperature difference between the evaporator and ambient. This is determined by the UA value of the evaporator, for which that of the other heat exchanger is used as a reference.

\section{F. Discharging}

The cycle points are the same, and denoted with a prime after the number to signify discharge (e.g. $2^{\prime}$ ).

The condenser has the same UA value as the evaporator since this is the same piece of equipment, again determining the temperature difference relative to ambient. Point $4^{\prime}$ is set as saturated liquid. The pressure of point $3^{\prime}$, the inlet of the heat exchanger, is set by the input $p_{\text {ratio }}=p_{3^{\prime}} / p_{3}=1$, by default (the effect of varying this parameter will be studied later). The working fluid then goes through the heat exchanger, receiving heat from the storage fluid, and coming out at point $2^{\prime}$. It is then expanded to point $1^{\prime}$, at the same pressure as $4^{\prime}$, which may lie inside or outside the saturation curve. Finally, the working fluid is condensed from $1^{\prime}$ to $4^{\prime}$.

\section{G. Outputs}

Round-trip efficiency is the main measure of the cycle's performance and reflects how reversible it is:

$$
\chi=\tau \frac{w_{d i s}}{w_{c h}}=\tau \frac{\left(h_{2}-h_{1}\right)-\left(h_{3}-h_{4}\right)}{\left(h_{2^{\prime}}-h_{1^{\prime}}\right)-\left(h_{3^{\prime}}-h_{4^{\prime}}\right)}
$$

with $\tau=\dot{m}_{s} / \dot{m}^{\prime}{ }_{s}=t_{d i s} / t_{c h}$

Power density $\left(\mathrm{MW} / \mathrm{m}^{3} \cdot \mathrm{s}^{-1}\right)$, is also computed and gives an indication of the size of the turbomachinery required per unit power:

$$
P_{\text {den }}=\rho_{1} w_{\text {in }}
$$

$\rho_{1}$ is the lowest mass density of the working fluid in the cycle, at point 1 . The higher the power density, the more compact and therefore cheaper the turbomachinery can be.

Heat to work ratio includes all heat and work flows in the cycle:

$$
\mathrm{HWR}=\frac{\left|q_{h}\right|+\left|q_{c}\right|}{w_{i n}}=\frac{\left(h_{2}-h_{3}\right)+\left(h_{1}-h_{4}\right)}{\left(h_{2}-h_{1}\right)-\left(h_{3}-h_{4}\right)}
$$

The lower the heat to work ratio, the lower the total cost per unit power capacity of the heat exchangers is. This correlates with higher storage temperatures, since the heat will have higher exergy.

In addition to the above metrics, the model computes the breakdown of the exergetic losses by component. It also tracks the storage temperatures at charge and discharge. Higher temperatures will result in higher exergy density for a given material. Finally, the vapour fraction $x$ of the working fluid at points 4 and $1^{\prime}$ is measured, when these are inside the saturation curve. While some degree of wetness or dryness is acceptable [15], [16], going too deeply into the two-phase region can be problematic because the efficiency of the turbomachinery is reduced under two-phase flow and because the droplets of condensation will mechanically damage the turbomachinery. This means that storage at higher temperatures, for which the cycle tends to have superheating and thus avoid the two-phase region, will be favoured for practical purposes.

\section{RESULTS}

\section{A. Dependence on top temperature}

It is important to note that the following results are not independent of the assumption of bottoming at ambient temperature and the storage medium, since the losses in the heat exchanger (a substantial fraction of the overall losses) depend directly on the pairing with the working fluid. In theory, a given fluid's heat capacity variation could match that of one storage medium better than another's.

According to Fig. 5, only carbon dioxide can operate at a temperature compatible with unpressurised water. However, its peak efficiency is above $100^{\circ} \mathrm{C}$, so operating at the optimal point requires a different fluid (or water pressurised to around 2.5 bar). At 100 and $192^{\circ} \mathrm{C}$, the discontinuity on the graphs in Fig. 5 corresponds to the model switching discretely from one storage medium to another.

Figure 6 illustrates how the exergy loss breaks down in the cycle by component, with R13I1 as an example. For each fluid, the fraction of work lost initially decreases with increasing maximum storage temperature, because at lower temperatures, the working fluid comes closer to the critical point in the heat exchanger. Operating near the critical point often leads to high specific heat variation, which directly results in large temperature differences and thus entropy generation in the heat exchanger. Thus, at higher temperatures away from the critical point, heat capacity variation and entropy generation are reduced. However, if the temperature increases too much, the losses start to increase again due to a decrease in work ratio. 


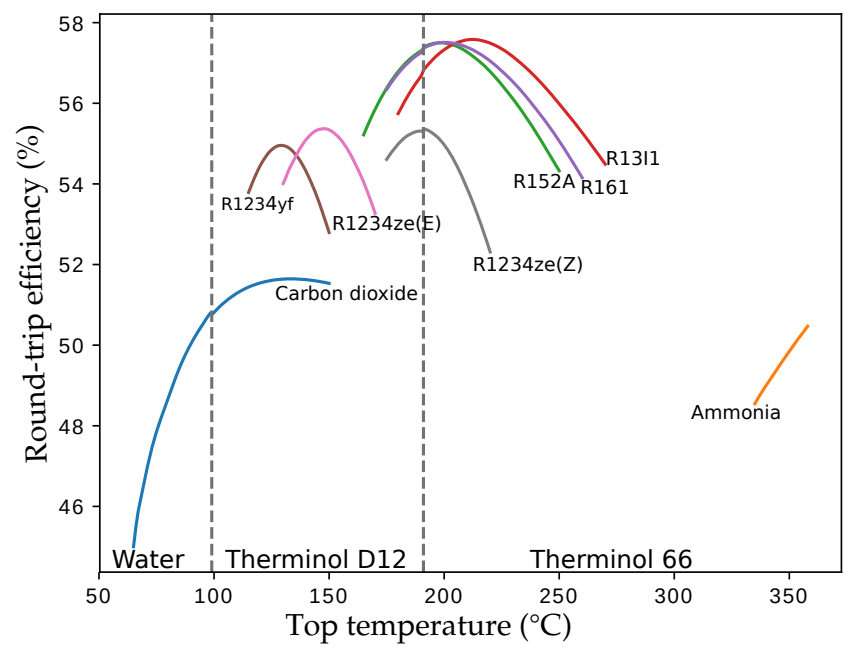

Fig. 5. Round-trip efficiency as a function of top temperature for each fluid. $\mathrm{NB}$ : some of the plots have been cut at the left and right ends for less overlap and more legibility. What matters most is the efficiency peak.

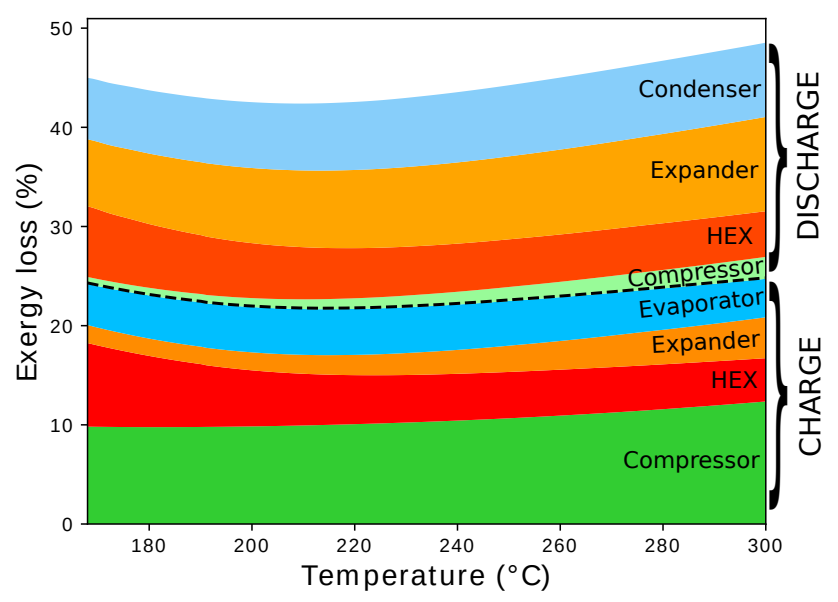

Fig. 6. Breakdown of relative exergy losses as a function of top temperature for R13I1

Work ratio is high at lower storage temperatures because compressing or expanding a liquid requires very little work. This can be understood from the fundamental thermodynamic relations $\delta w+\delta q=d h=T d s+v d p$. Applied for example to an adiabatic $(\delta q=0)$ and reversible (therefore isentropic, $d s=0)$ compression, the work applied is $\delta w=d p / \rho$. For the same pressure variation, a gas will have much lower density than a liquid and therefore require more work to compress. For a higher $T_{2}, \rho_{2}$ becomes closer to $\rho_{3}$ and therefore the work ratio is closer to 1 .

The work ratio varies greatly from one fluid to the next, as shown in Table IV. Turbomachinery losses decrease with higher work ratios, and therefore are low at lower storage temperatures. Because of the differences in work ratio, some fluids will perform much better than others even with the same given polytropic efficiency. As the storage temperature
TABLE IV

OPERATING POINTS THAT MAXIMISE ROUND-TRIP EFFICIENCY

\begin{tabular}{lccccc}
\hline Fluid & $\begin{array}{c}T_{2} \\
{ }^{\circ} \mathrm{C}\end{array}$ & $\begin{array}{c}T_{s} \\
{ }^{\circ} \mathrm{C}\end{array}$ & $\begin{array}{c}\text { Pressure } \\
\text { bar }\end{array}$ & Work ratio & $\begin{array}{c}\chi \\
\%\end{array}$ \\
\hline R1234yf & 129 & $36-128$ & $4-58$ & 8.2 & 55.0 \\
Carbon dioxide & 134 & $43-126$ & $46-207$ & 3.4 & 51.6 \\
R1234ze(E) & 147 & $38-146$ & $3-63$ & 9.6 & 55.4 \\
R1234ze(Z) & 191 & $46-189$ & $1-59$ & 14.7 & 55.4 \\
R152A & 198 & $43-193$ & $4-100$ & 9.9 & 57.5 \\
R161 & 200 & $44-195$ & $6-124$ & 8.4 & 57.5 \\
R1311 & 212 & $45-206$ & $3-83$ & 9.4 & 57.6 \\
Ammonia & 358 & $47-294$ & $5-158$ & 6.0 & 50.5 \\
\hline
\end{tabular}

TABLE V

ADDITIONAL CHARACTERISTICS OF OPTIMAL OPERATING POINTS

\begin{tabular}{lcccl}
\hline Fluid & $x_{4}$ & $x_{1^{\prime}}$ & $p_{2} / p_{1}$ & Flammability \\
\hline R1234yf & $20 \%$ & $94 \%$ & 13 & Flammable \\
Carbon dioxide & $27 \%$ & superheat & 5 & Non flammable \\
R1234ze(E) & $21 \%$ & $95 \%$ & 20 & Slightly flammable \\
R1234ze(Z) & $21 \%$ & $98 \%$ & 61 & Slightly flammable \\
R152A & $22 \%$ & superheat & 28 & Flammable \\
R161 & $22 \%$ & superheat & 22 & Flammable \\
R13I1 & $22 \%$ & superheat & 28 & Non flammable \\
Ammonia & $43 \%$ & superheat & 32 & Slightly flammable \\
\hline
\end{tabular}

increases, work ratio falls and associated losses rise.

Due to the tradeoff between losses in the heat exchanger and turbomachinery respectively, the round-trip efficiency peaks at a certain temperature in Fig. 5. Table IV details the operating conditions of the peaks. Clearly, fluids with high total roundtrip efficiency are preferable, but a secondary consideration is how "sharp" the efficiency peak is: a fluid with a wider peak will enable a more manageable margin of operation, i.e. deviating from the exact optimal temperature will not result in a significant efficiency penalty. Overall, the fluids have similar peaks in efficiency. Note that the temperature range of Therminol 66 is too limited to draw any significant conclusions as to the optimal performance of ammonia: it would work better at storage temperatures beyond the boiling point of Therminol 66, with molten salts for example.

\section{B. Pareto fronts and rankings}

The heat to work ratio and power density are also important, and their dependence on temperature is quite clear: the former decreases with $T_{2}$ whilst the latter increases, both fairly linearly. It is impossible to maximise performance in terms of round-trip efficiency, power density, and heat to work ratio all at the same time. Therefore, a Pareto front was constructed of the fluids' power density and heat to work ratio at the optimal operating temperature for round-trip efficiency (Fig. 7). An ideal fluid would be situated in the bottom right corner: low heat exchanger cost and low turbomachinery cost, per unit power. These vary quite significantly from one fluid to the next, with carbon dioxide and ammonia dominating in these respects, although their efficiencies are the lowest among the fluids.

As shown in Table $\mathrm{V}$, at their optimal operating temperature, most fluids do not enter the two-phase region during discharge, except slightly the three wet fluids, which is a drawback for 


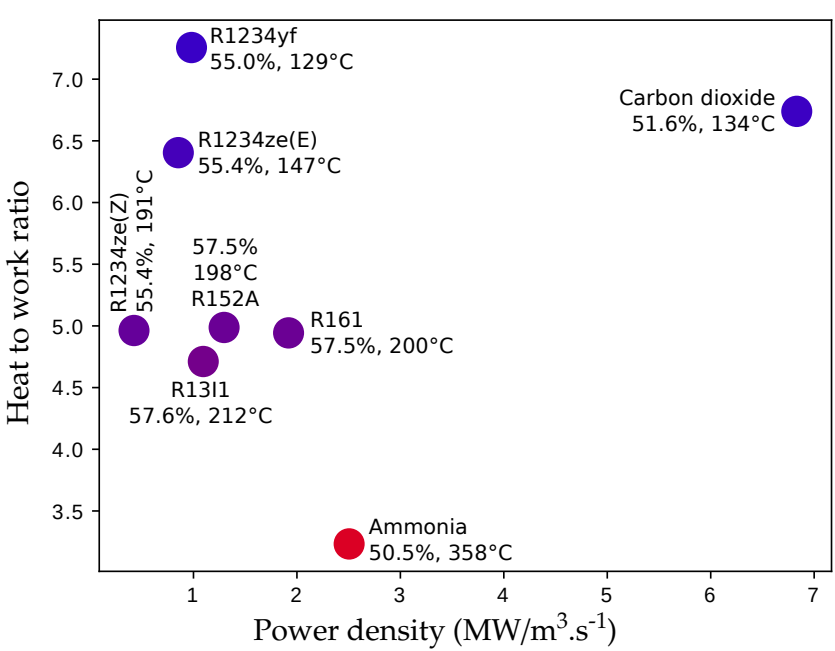

Fig. 7. Pareto front of HWR versus power density, with $\chi$ and $T_{2}$. The colour of the points reflects $T_{2}$ (from blue for low to red for high).

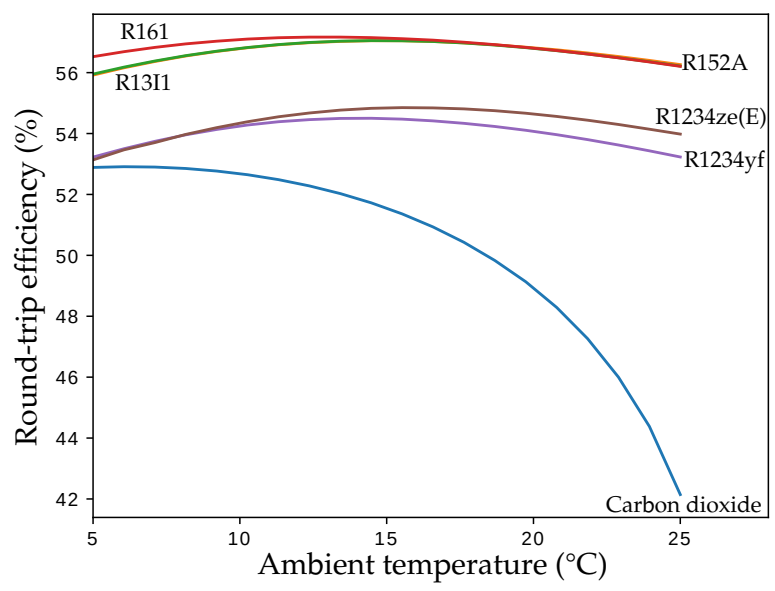

Fig. 8. Influence of ambient temperature on maximum round-trip efficiency

them. However, all the fluids do so during charge, so the liquid expander between points 3 and 4 will have to be able to deal with the presence of vapour; this is especially the case for ammonia. Another practical consideration is the value of $p_{2} / p_{1}$ : if this is low (around 4), then compression and expansion can be done in a single centrifugal stage, which lowers the turbomachinery cost. Carbon dioxide is attractive in this regard; R1234ze(Z) on the other hand is not, while the rest of the fluids are in a range around 20.

\section{Sensitivity analysis}

The cycle bottoms at ambient temperature i.e. the environment acts as the cold store. The performance of the system is therefore affected by ambient conditions. This is quantified in Fig. 8, which shows the influence of $T_{0}$ on round-trip efficiency. The value of $p_{2} / p_{1}$ from the design point of $T_{0}=15^{\circ} \mathrm{C}$ is used (see Table V), because the pressure ratio of

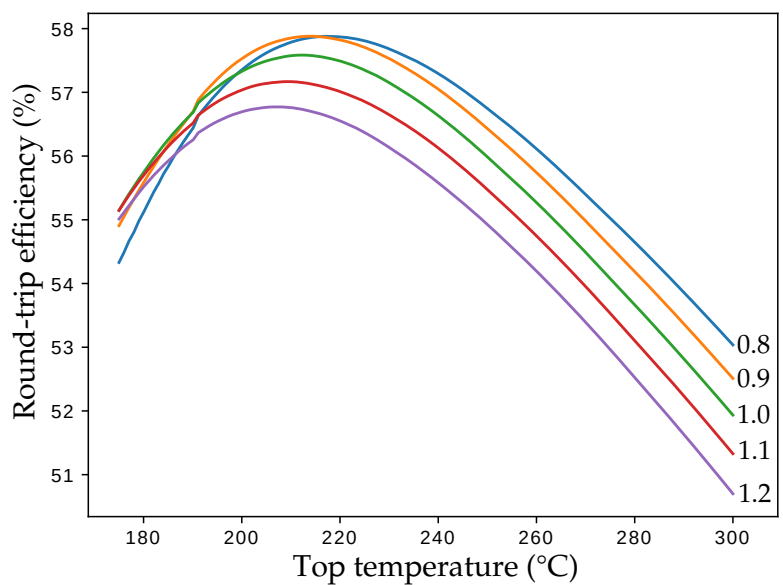

Fig. 9. Round-trip efficiency of R13I1 for different values of $p_{\text {ratio }}=$ $p_{3^{\prime}} / p_{3}$

the turbomachinery in a real system would remain relatively constant. $T_{0}$ is varied from $5^{\circ} \mathrm{C}$ to $25^{\circ} \mathrm{C}$. Ammonia was not included in this sensitivity study, since its optimal operating point lies outside the range of values for $T_{2}$.

The maximum round-trip efficiency for most of the fluids is largely unaffected by $T_{0}$ (around $1 \%$ point variation overall), which means they can be used year-round without a reduction in performance. Carbon dioxide is the only exception: because $T_{\text {crit }}$ is so close to $T_{0}$, it is very sensitive to ambient conditions (around 10\% points of variation overall), which is a substantial drawback.

\section{Focus on R13I1}

R13I1 represents a compromise on all metrics: it achieves the highest efficiency, second-lowest heat to work ratio, and average power density. R161 has similar performance with higher power density, but is less safe, as shown in Table V. R13I1 has therefore been chosen to study the effect of varying the value of $p_{\text {ratio }}=p_{3^{\prime}} / p_{3}$.

A value of $p_{\text {ratio }}<1$ i.e. lowering the top pressure during discharge tends to increase the work ratio and reduce turbomachinery losses. The round-trip efficiency then increases because the output work has increased, for the same input work. However, the gain is modest, around $0.3 \%$ points, as can be seen in Fig. 9. Furthermore, if the pressure ratio is lowered too much, then the heat exchange process during discharge will come too close to the critical point and losses will increase again, for the reasons explained in previous sections. This can be seen in Fig. 9, where there is no gain at the peak efficiency in lowering $p_{\text {ratio }}$ from 0.9 to 0.8 , as well as at the low end of temperatures, where smaller pressure ratios result in worse efficiency.

A value of $p_{\text {ratio }}>1$ i.e. increasing the top pressure during discharge is undesirable because it lowers the work ratio and therefore increases turbomachinery losses. The only advantage is to reduce heat exchanger losses by moving away from 
the critical point, but this is less pronounced when the cycle operates near the optimal top temperature, and so round-trip efficiency is lower than for $p_{\text {ratio }}=1$, as seen in Fig. 9.

\section{CONCLUSIONS}

The presented cycle model provides a useful comparison of the performance of various working fluids for transcritical cycles, and characterises the storage temperature which maximises round-trip efficiency. Additional objectives of high power density and low heat to work ratio were also considered and Pareto fronts were used to further rank the fluids by performance according to these two metrics.

Carbon dioxide is found to achieve high power density as expected from the literature, but performs at lower round-trip efficiency compared to other fluids and is much more sensitive to ambient temperature. It also has an undesirably high heat ratio because of its low storage temperature. However, this comes with the benefit of being able to operate at temperatures compatible with unpressurised water storage, which could be economically attractive. Overall, R13I1 represents a good compromise between the performance measures and safety.

Further work could explore different storage fluids, such as high temperature molten salts, which would match well with an ammonia cycle. An economic analysis could further differentiate between the fluids, notably by examining the tradeoff between the additional cost of having intermediate storage tanks versus the associated reduction in heat exchanger losses. The influence of ambient temperature could be further investigated by differentiating between charge and discharge to see whether a day/night cycle scheduling is advantageous.

\section{NOMENCLATURE}

$\begin{array}{ll}\text { Acronyms } \\ \text { CAES } & \text { Compressed air energy storage } \\ \text { PHS } & \text { Pumped hydro storage } \\ \text { PTES } & \text { Pumped thermal energy storage } \\ \text { Symbols } & \\ \chi & \text { Round-trip efficiency }(\%) \\ \dot{m} & \text { Mass flowrate }(\mathrm{kg} / \mathrm{s}) \\ \rho & \text { Working fluid mass density }\left(\mathrm{kg} / \mathrm{m}^{3}\right) \\ \tau & \text { Ratio of discharge over charge time } \\ c_{p} & \text { Isobaric specific heat capacity }(\mathrm{J} / \mathrm{kg} / \mathrm{K}) \\ h & \text { Specific enthalpy }(\mathrm{J} / \mathrm{kg}) \\ p & \text { Pressure (bar) } \\ p_{\text {ratio }} & \text { Pressure ratio between charge } / \mathrm{discharge} \\ q & \text { Specific heat transfer }(\mathrm{J} / \mathrm{kg}) \\ T_{0} & \text { Ambient temperature }(\mathrm{K}) \\ T_{s} & \text { Temperature of the charged storage medium }\left({ }^{\circ} \mathrm{C}\right) \\ T_{t o p} & \text { Top temperature in the charge cycle }(\mathrm{K}) \\ w & \text { Specific work }(\mathrm{J} / \mathrm{kg}) \\ x & \text { Vapour fraction }\end{array}$

\section{REFERENCES}

[1] A. White, G. Parks, and C. N. Markides, "Thermodynamic analysis of pumped thermal electricity storage," Applied Thermal Engineering, vol. 53, no. 2, pp. 291-298, 52013.
[2] M. Abarr, B. Geels, J. Hertzberg, and L. D. Montoya, "Pumped thermal energy storage and bottoming system part A: Concept and model," Energy, vol. 120, pp. 320-331, 22017.

[3] M. Mercangöz, J. Hemrle, L. Kaufmann, A. ZGraggen, and C. Ohler, "Electrothermal energy storage with transcritical CO2 cycles," Energy, vol. 45, no. 1, pp. 407-415, 92012.

[4] Y.-J. Baik, J. Heo, J. Koo, and M. Kim, "The effect of storage temperature on the performance of a thermo-electric energy storage using a transcritical CO2 cycle," Energy, vol. 75, pp. 204-215, 102014.

[5] M. Morandin, M. Mercangöz, J. Hemrle, F. Maréchal, and D. Favrat, "Thermoeconomic design optimization of a thermo-electric energy storage system based on transcritical CO2 cycles," Energy, vol. 58, pp. 571-587, 92013.

[6] F. Ayachi, N. Tauveron, T. Tartière, S. Colasson, and D. Nguyen, "Thermo-Electric Energy Storage involving $\mathrm{CO} 2$ transcritical cycles and ground heat storage," Applied Thermal Engineering, vol. 108, pp. 14181428, 92016.

[7] M. Morandin, F. Maréchal, M. Mercangöz, and F. Buchter, "Conceptual design of a thermo-electrical energy storage system based on heat integration of thermodynamic cycles Part A: Methodology and base case," Energy, vol. 45, no. 1, pp. 375-385, 92012.

[8] Y. Ahn, S. J. Bae, M. Kim, S. K. Cho, S. Baik, J. I. Lee, and J. E. Cha, "Review of supercritical $\mathrm{CO} 2$ power cycle technology and current status of research and development," Nuclear Engineering and Technology, vol. 47, no. 6, pp. 647-661, 102015.

[9] W. H. Stein and R. Buck, "Advanced power cycles for concentrated solar power," Solar Energy, vol. 152, pp. 91-105, 2017.

[10] Linde Gases, "Refrigerants Environmental Data. Ozone Depletion and Global Warming Potential."

[11] United Nations Environment Programme, "HCFC blends."

[12] G. Restrepo, M. Weckert, R. Brüggemann, S. Gerstmann, and H. Frank, "Ranking of refrigerants," Environmental Science and Technology, vol. 42, no. 8, pp. 2925-2930, 42008.

[13] Australian Department of the Environment and Energy, "HFC global warming potential," Australian Department of the Environment and Energy, Tech. Rep.

[14] G. Nellis and S. Klein, Heat transfer, S. A. Klein, Ed. Cambridge: Cambridge : Cambridge University Press, 2009., 2009.

[15] J. Bao and L. Zhao, "A review of working fluid and expander selections for organic Rankine cycle," Renewable and Sustainable Energy Reviews, vol. 24, pp. 325-342, 82013.

[16] O. Demuth, "Preliminary assessment of condensation behavior for hydrocarbon-vapor expansions which cross the saturation line near the critical point," Idaho National Engineering and Environmental Laboratory, Idaho Falls, ID, Tech. Rep., 71982. 